\title{
JADE: A Tool for Rapid Crisis Action Planning
}

\author{
Alice M. Mulvehill \\ BBN Technologies -- A Part of GTE Corporation \\ 10 Moulton Street \\ Cambridge, MA 02138 \\ 617-873-2228 \\ amm@bbn.com \\ Joseph A. Caroli \\ Air Force Research Lab/IFTB \\ 525 Brooks Rd. \\ Rome NY 13441 \\ 315-330-4205 \\ carolij@rl.af.mil
}

\begin{abstract}
The Joint Assistant for Deployment and Execution (JADE) offers a new technique for rapid force deployment planning, especially in crisis situations. JADE is being developed by BBN Technologies under contract to the Air Force Research Lab and the Defense Advanced Research Projects Agency (DARPA). It is being designed to operate within the next generation Global Command and Control System. JADE implements state of the art technology to reduce the time typically required for building a Time Phased Force Deployment Data package, which presently takes days or weeks, down to approximately one hour. In order to support the command and control needs of planners today and in the twenty-first century, several challenges are being met to transition JADE from a research prototype to a fully operational system. These include integrating various data systems, seeking user input and buy-in, porting to new computing environments, assuring compliance with the Defense Information Infrastructure Common Operating Environment, and testing in exercise scenarios. A potential technology transition candidate for JADE is the Adaptive Course of Action (ACOA) program. ACOA is a DARPA advanced conceptual technology demonstration (ACTD) that provides the means to employ new technologies in the Joint Operations Planning and Execution environment. The ACOA tool suite is also a key enabler in achieving Joint Vision 2010. In this paper, a detailed overview of JADE is provided along with plans for technology transition.
\end{abstract}

\subsection{JADE Overview}

JADE demonstrates breakthrough technology that enables a military planner to build a force deployment plan, including a Time Phased Force Deployment Data (TPFDD) package in less than one hour. JADE is a knowledge-intensive planning technique that employs case-based and generative planning methods to handle large-scale, complex plans in minimal time. The tool is capable of rapid retrieval and reuse of previous plan elements and employs an easy to use maporiented drag and drop interface. Force modules from previous plans whose force capabilities and composition satisfy the current situation can be dragged from the plan library (casebase) and 
dropped onto a geographic destination. Constraint checking is conducted during plan development and the user is automatically reminded of ways to modify and improve the plan in real time. JADE provides advanced capabilities for military planners, and a solution to the challenge of rapidly producing force deployment plans and TPFDDs.

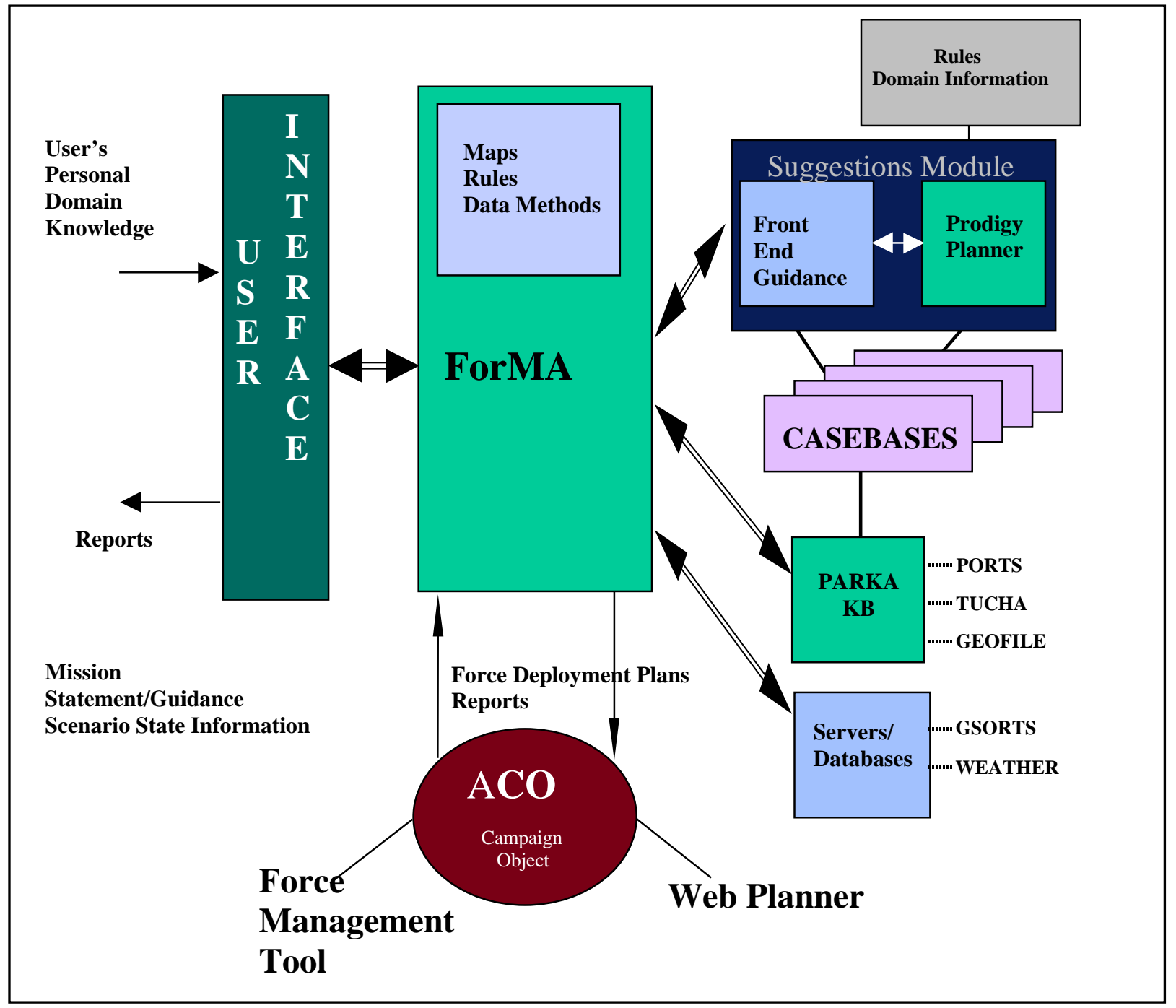

Figure 1 -- JADE Architecture

The current architecture of JADE is presented in Figure 1. JADE's three major technology components (ForMAT, Prodigy, and PARKA) support the user in modifying force compositions, describing force capabilities, and in tailoring the evolving force deployment plan to changing mission requirements. To date JADE's plan repository (casebase) is comprised of 4 different casebases that support: training, general planning, the ACOA environment, and the JEFX (Joint Expeditionary Force Experiment) environment. Each casebase is comprised of some number of TPFDDs and their constituent Force Modules and Unit Line Numbers. For example, the general 
casebase contains 33 TPFDDs, 431 Force Modules, and 17,169 Unit Line Numbers (ULNs). Plans in this repository support both crisis action and deliberate planning.

\subsection{ForMAT}

Users interact with JADE through a user interface that is directly connected to the ForMAT (Force Management and Analysis Tool) module. ForMAT [Mulvehill, 1995] provides an environment for building force modules (FMs). A FM describes a force or set of forces that can be used to satisfy some mission requirement. Typically a FM is a grouping of combat, combat support, and combat service support forces, and ranges in size from the smallest combat element to the largest combat element. It may specify accompanying supplies and the required movement resupply and personnel necessary to sustain forces for a minimum of 30 days. [AFSC Pub, 1997] FMs can be linked with other FMs to create a TPFDD. In JADE, the user can explicitly specify hierarchical relationships among FMs (as displayed in Figure 2 where FMs $A, B, C$, and $D$ are forces that comprise the parent FM (NEO). ForMAT saves information about FMs and how they are used in a data repository called a casebase. ForMAT employs case-based reasoning (CBR) technology to enable a user to make use of past experience (past planned FMs) to rapidly solve new problems. For the joint command and control situation, individual casebases may be used to represent the planning particular to specific theaters and to the joint environment.

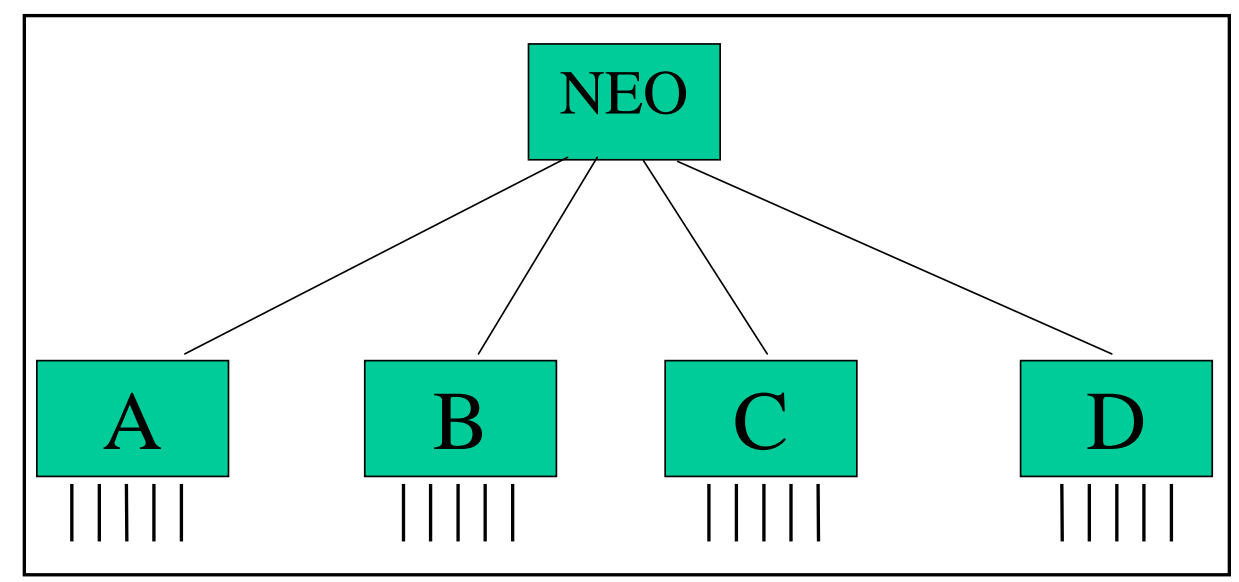

Figure 2 -- FM Hierarchy

ForMAT also provides the user with tools for linking the data of the TPFDD to the mission that the deployment forces are intended to support and for describing what capabilities the forces contain that enable them to support a mission. Mission guidance can simply be stored as a file or it can be derived from a campaign development tool. For example, in Figure 1, mission information is obtained electronically by ForMAT from the ACOA campaign object where the campaign planning elements are stored. It is this linking of the forces to the mission that extends the TPFDD into a Deployment Plan.

Given mission guidance, and a plan library (casebase) of previously developed or executed deployment plans, along with data from other databases such as GSORTS (see Table 2), a user can quickly construct a new deployment plan or modify an existing one. The JADE interface 
enables users to construct a new plan by simply dragging one or more FMs from the casebase directly to a location on a map where it is to be deployed. Guidance is provided to the user on how FMs need to be tailored to fit the new mission. This guidance is provided through ForMAT's link to the Prodigy system.

\subsection{Prodigy}

Prodigy [Veloso et al., 1997] is a multi-strategy planning and learning architecture that can solve planning problems in a number of different ways. One way is with the Prodigy4.0 module that employs a state-space nonlinear planner and follows a means-ends analysis backward-chaining search procedure that reasons about both multiple goals and multiple alternative operators from its domain theory as they are appropriate for achieving such goals. A domain theory is composed of a hierarchy of object classes and a suite of operators and inference rules that change the state of the objects. A planning problem is represented by an initial state (objects and propositions about the objects) and a set of goal expressions to achieve. Planning decisions consist of choosing a goal from a set of pending goals, choosing an operator (or inference rule) to achieve a particular goal, choosing a variable binding for a given operator, and deciding whether to commit to a possible plan ordering and to get a new planning state or to continue subgoaling for unachieved goals. Different choices give rise to different ways of exploring the search space.

Another strategy available to Prodigy is the case-based replay mode. In this mode, Prodigy uses analogy to create plans, interpret and store planning episodes, and retrieve and reuse multiple past plans that are found similar to new problems. Stored plans are annotated with plan rationale, and reuse involves adaptation driven by the plan rationale.

In JADE, we developed a Front End that links ForMAT to the Prodigy planner. The Front End collects information such as mission goals and state information about the planning context through ForMAT and presents it to Prodigy for use in planning. Prodigy then provides guidance through the Front End to the Suggestions Module which then presents guidance and/or suggestions to the user about how to modify or create a deployment plan. For example, when the user is initially looking for FMs that will satisfy new mission requirements, Prodigy will use its own information about previous plans to make a suggestion about what particular FMs should be used, or what entire past plans are most similar to the current mission situation. The Suggestions Module will present this information, as well as information from ForMAT to remind the user that forces are to be deployed to specific geographical locations and when certain forces are preferred for inclusion within a deployment plan.

Because the Prodigy system uses mission goals and causal information, the resulting plan is in a strategy-to-task framework. That is, the steps in the deployment plan are related to the causal reasons for which the forces are to be used. Although Prodigy is capable of generating the entire deployment plan, in JADE Prodigy can only provide suggestions to the user who has the ultimate decision in what gets deployed to where and when.

\subsection{PARKA}

Another component of the JADE architecture is the PARKA knowledge base (PARKA-KB). PARKA-KB is a high performance knowledge base management system. Although it is similar 
to a relational database, it uses technology that supports and takes advantage of data hierarchies. It has been shown to effectively handle extremely complex "structure matching" queries against large knowledge bases [Hendler and Mulvehill, 1997].

In JADE, several PARKA KBs are used to support plan retrieval and the retrieval of the associated data (see Table 2) that support deployment plan generation. PARKA-KBs store the TPFDDs (both FMs and ULNs); a representation of the country code information, referred to as the "GEOFILE" database; a representation of seaports (PORTS) data; and Type Unit Characteristics (TUCHA) information. Using these PARKA-KBs, the JADE user can retrieve the desired FMs, ULNs, or information about the geographical location that forces are to be deployed to.

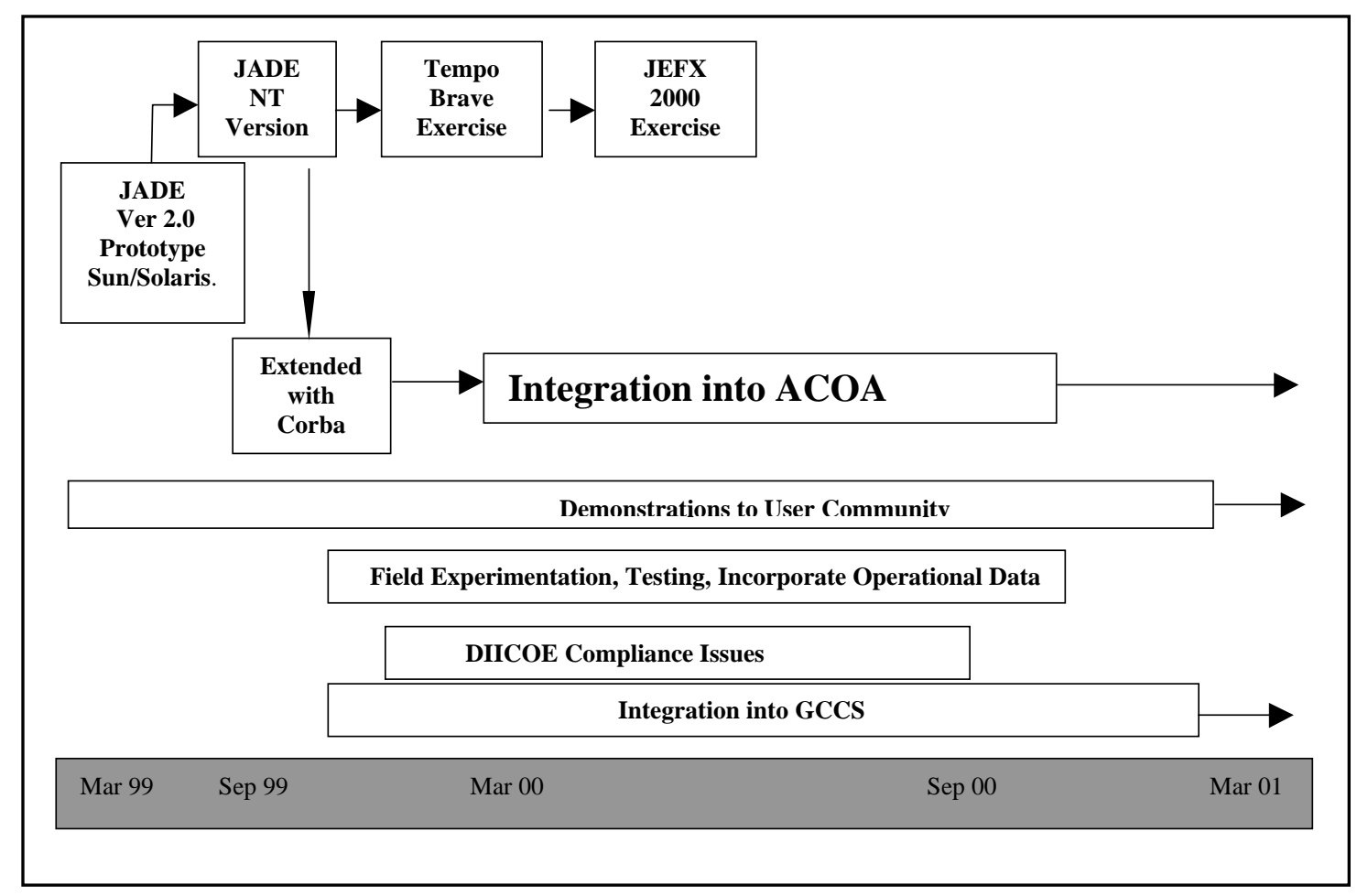

Figure 3 -- Timeline View of JADE Technology Transition Activities

\subsection{Technology Transition of JADE}

JADE is a prototype residing on both NT and UNIX-based platforms at BBN Technologies and the Air Force Research Lab. A major challenge facing the development team is how to transition the software to an operational setting. End users of JADE will be those responsible for planning the force deployment activities of joint military campaigns. This responsibility lies in the hands of the combatant commanders and is conducted in close coordination with the United States Transportation Command. The operational tools now used for managing force deployment planning include the JOPES (Joint Operation Planning and Execution System) Editing Tool (JET), Rapid Query Tool (RQT), Joint Flow Analysis Tool for Transportation (JFAST) and 
others. Readers interested in more detail should see [Joint Pub 1993]. Figure 3 illustrates a timeline view of the activities required in order for JADE to support the end users.

\subsection{Technology Transition Activities}

In an effort to elicit user support the most current version of JADE works within the NT environment. (Note: The first two versions of JADE prototypes were developed in a UNIXbased Sun Solaris environment). Before JADE is fielded, the targeted end users must be confident that it will adequately support force deployment planning for joint military operations. JADE participated as part of ACOA in the 1999 Tempo Brave exercise and we plan to use the JEFX 2000 exercise as another testing forum. Also, we have sought to facilitate technology development through numerous demonstrations of the software. As illustrated in Table 1, an aggressive demonstration initiative has taken place in the past year. The table lists organizations receiving demonstrations on an individual basis.

\begin{tabular}{|c|c|}
\hline $\begin{array}{l}\text { + US Pacific Command } \\
\text { + US Central Command } \\
\text { + US Southern Command } \\
\text { + US Transportation Command } \\
\text { + DARPA ACOA Program Office } \\
\text { + DARPA/DISA Joint Theater Logistics } \\
\text { Advanced Concept Technology Demo } \\
\text { Office } \\
\text { + Naval Operations Strategic Studies } \\
\text { Group } \\
\text { + Air Force Scientific Advisory Group } \\
\text { + Air Force Chief Scientist } \\
\text { DoD Technical Area Review and } \\
\text { Assessment Team (TARA) }\end{array}$ & $\begin{array}{l}\text { + Air Force Material Command } \\
\text { + Air Mobility Command } \\
\text { + Air Force Information Warfare Office } \\
\text { + International Technology Cooperation } \\
\text { Program Committee } \\
\text { + Air Force Command \& Control Sector } \\
\text { + Air Force Future Concept } \\
\text { Development Group } \\
\text { + Air Force Research Lab Human } \\
\text { Effectiveness Directorate } \\
\text { + JOPES User Advisory Group } \\
\text { + Air Force Director of Command \& } \\
\text { Control }\end{array}$ \\
\hline
\end{tabular}

Table 1 -- Organizations Receiving JADE Demonstrations

Due to security considerations, the prototype is now populated with unclassified exercise and other hypothetical data. Effort is underway to draw live operational data into JADE. Table 2 describes the data systems that need to be accessed by JADE. Many of these systems are resident in the Joint Operation Planning and Execution System automated data processing (JOPES ADP) environment. An experimental facility has been established at the Air Force Research Lab in Rome NY to begin utilizing JOPES ADP data within JADE.

During the Tempo Brave exercise JADE moved from a lab environment to its maiden operational usage. To support the exercise, JADE was installed in operational environments at several sites and the casebase was populated with a set of TPFDDs. A few selected users were trained to make use of the library to support both deliberate and crisis planning. During Tempo Brave, JADE acquired mission requirements from the ACOA Campaign Object to aide TPFDD development. Under the control of a trained operator, JADE was successfully used to develop 
and edit deployment plans. Feedback from Tempo Brave is currently being used to improve JADE -- specifically to support Level-4 detail refinement of force deployment requirements.

\begin{tabular}{|c|c|}
\hline Data System Name & Description \\
\hline $\begin{array}{l}\text { APORTS } \\
\text { Aerial Ports and Air Operating Bases File }\end{array}$ & $\begin{array}{l}\text { Contains airfield factors including: } \\
\text { - } \quad \text { throughput capacities } \\
\text { - } \quad \text { runway length and width } \\
\text { - } \quad \text { weight bearing capacity } \\
\text { - } \quad \text { aircraft parking space } \\
\text { - } \quad \text { fuel \& cargo storage capacity }\end{array}$ \\
\hline $\begin{array}{c}\text { PORTS } \\
\text { Port Characteristics }\end{array}$ & $\begin{array}{l}\text { Contains seaport factors including: } \\
\text { - } \quad \text { size and depth } \\
\text { - } \quad \text { number of berths } \\
\text { - } \quad \text { beach data } \\
\text { - } \quad \text { cargo handling \& storage capacity }\end{array}$ \\
\hline $\begin{array}{l}\text { FM Library } \\
\text { Force Module Library }\end{array}$ & $\begin{array}{l}\text { Force and sustainment information for joint combat, } \\
\text { combat support, and combat service support forces. }\end{array}$ \\
\hline $\begin{array}{c}\text { TUCHA } \\
\text { Type Unit Data File or } \\
\text { Type Unit Characteristics File }\end{array}$ & $\begin{array}{l}\text { Describes unit characteristics including: } \\
\text { - } \quad \text { number of personnel } \\
\text { - } \quad \text { number of personnel requiring transportation } \\
\text { - } \quad \text { types of cargo in unit } \\
\text { - weight and volume of equipment \& supplies }\end{array}$ \\
\hline $\begin{array}{c}\text { GEOFILE } \\
\text { Standard Geographic Locations }\end{array}$ & $\begin{array}{l}\text { Repository of military and other locations needed } \\
\text { during joint operation planning such as: } \\
\text { - military and commercial airports } \\
\text { - } \quad \text { seaports } \\
\text { - military bases }\end{array}$ \\
\hline $\begin{array}{l}\text { GSORTS } \\
\text { Global Status of Resources } \\
\text { and Training System }\end{array}$ & $\begin{array}{l}\text { Unit readiness information including: } \\
\text { - } \quad \text { personnel strength (actual and authorized) } \\
\text { - } \quad \text { percent of assigned equipment ready for operation }\end{array}$ \\
\hline
\end{tabular}

Table 2 -- Required Data Sources for JADE. [AFSC Pub, 1997]

\subsubsection{COE Compliance}

New and legacy systems incorporated into the Global Command and Control System (GCCS) must be compliant with the Defense Information Infrastructure Common Operating Environment 
(DII-COE). Since JADE's migration path is moving towards the GCCS, it needs to be DII-COE compliant. The Defense Information Infrastructure (DII) is a network of communications nodes, computers, weapon system interfaces, data systems, and other hardware and software systems that will meet the future information processing needs of the Department of Defense. The purpose of DII-COE is to standardize an architecture and a common operating environment (COE) that supports interoperability across the diverse components on the DII. Though evolving, there currently exists a set of procedures that are intended to provide guidance for developers to follow to assure such interoperability.

\subsection{The Vision for a Fielded JADE}

The vision is for JADE to become a component of the GCCS. GCCS currently performs many important command and control mission functions including force deployment planning. JOPES tools within GCCS are now used for both deliberate and crisis action planning, including TPFDD generation. While JOPES tools are widely used by the Joint Planning and Execution Community (JPEC), the speed at which TPFDDs are generated is a shortcoming noted by operational users. Additionally, current TPFDDs do not provide explicit links to the course of action (COA).

More efficient deployment planning tools are required to better support crisis planning and execution. While JOPES tools in GCCS are currently used to describe and manage the combat, combat support, and combat service support elements that are required in order to support a mission; the Pacific Command (PACOM), through the DARPA ACOA program, intends to move beyond JOPES tools. Their vision is to design an agile operations planning and execution system that is easy and intuitive to use and that quickly provides required information in support of time sensitive planning.

\section{1 $A C O A$}

The Adaptive Course of Action (ACOA) program provides a near real-time distributive collaborative environment that supports end-to-end joint planning by multiple participants including the supported CINC, supporting CINCs, and the commander of the Joint Task Force (CJTF) (See Figure 4). Through the use of collaborative and joint decision support tools, planners and operators are able to share a dynamic workspace for situational assessment and to rapidly adapt to situational changes.

The principal Government sponsors of the ACOA-ACTD include PACOM and the DARPA/DISA (Defense Advanced Research Projects Agency/Defense Information Systems Agency) Advanced Information Technology Services Joint Program Office. A major goal of the program is to cut crisis planning time in half. Both commercial-off-the-shelf and government developed emerging information technologies are being applied to meet the goal. The ACOA mission is stated as "develop and demonstrate advanced technology enabling geographically dispersed planning and executing forces to rapidly generate, assess, and adapt courses of action for military operations" [Jensen, 1999]. The initiative is viewed as a dramatic reengineering of the JOPES process and not just a marginal improvement or fine-tuning. ACOA is being designed to significantly improve both planning and execution activities at all levels including unit, tactical, operational, theater, and strategic. 


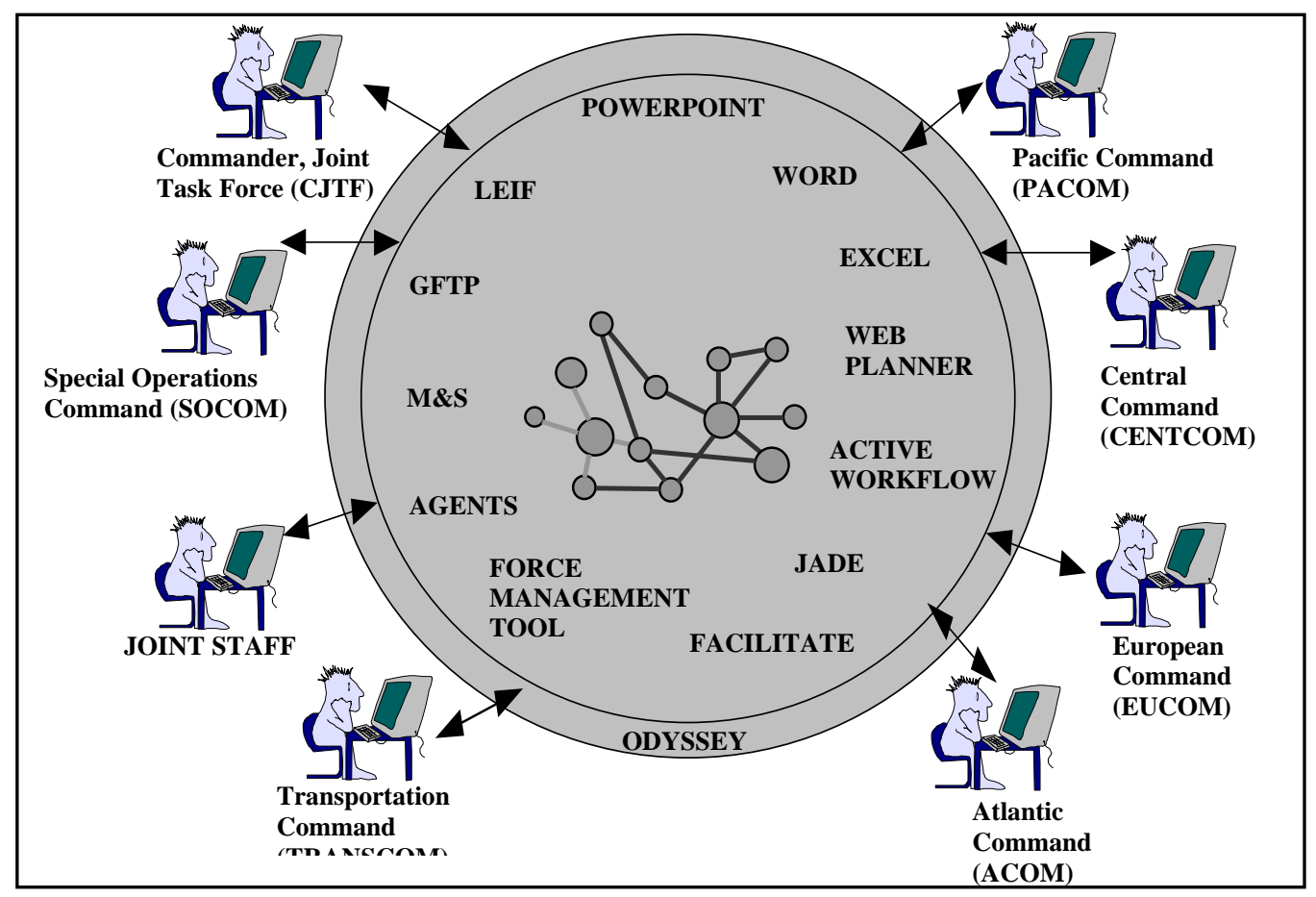

Figure 4 -- The ACOA Environment ${ }^{1}$

Some of the ACOA tools provide step-by-step guidance for planners involved in joint planning, and some ACOA tools generate products at various stages of planning and provide analysis capabilities to enable the planners to test alternative planning strategies. For example, there are several tools in ACOA that are oriented toward supporting COA and force planning. Two of these tools are the WebPlanner and JADE. The WebPlanner extends the ability of joint planning tools and information to all members of the joint planning team. It accelerates multi-staff collaboration during situation assessment, execution planning, and execution. It provides for the generation of planning products through its tight integration with Microsoft Word and PowerPoint. Finally, the WebPlanner provides an automated assistant that guides novice users through the critical steps of plan development.

In ACOA, JADE works with other tools like the WebPlanner to develop the overall campaign. For example, the WebPlanner provides JADE with information about the developing mission/situation through its Word documents and about the forces that are preferred for use during an operation. JADE then is used to develop the supporting deployment plan. Through JADE, a user has the ability to save a deployment plan to the ACOA campaign object and to locally save previously executed plans and hypothetical force compositions. Plans can then be reused when needed to support the rapid construction of an $80 \%$ deployment solution to a given situation. This ability to use past experience to rapidly build new plans also gives the ACOA

\footnotetext{
${ }^{1}$ LEIF - Light Weight Extensible Information Framework, GFPT - Geospacial Force Planning Tool, M \& S - Movement and Scheduling
} 
CINC the ability to quickly analyze the costs and benefits associated with using different force compositions to achieve mission goals.

\subsection{Summary}

We believe that JADE will contribute to achieving Joint Vision 2010 since JADE focuses on solving a dilemma which has been confronting force deployment planners for years, i.e., rapid TPFDD generation with explicit links to the mission objectives. The authors of this paper feel that incorporating JADE into the ACOA environment and by having JADE participate in joint exercises like JEFX2000 will result in better tools that will, in the long run, enable military planners, e.g., the CINC to better determine how best to use forces to support their mission objectives. JADE will also enable force deployment planners to better match force capability with mission requirements and determine the transportation feasibility of their force deployments in support of a particular mission.

\subsection{Bibliography}

[Joint Pub, 1993] Joint Operation Planning and Execution System, Joint Pub 5-03.1, Office of the Chairman, The Joint Chief s of Staff, Washington DC, 1993

[AFSC Pub, 1997] The Joint Staff Officer's Guide, AFSC Pub 1, Armed Forces Staff College, 1997.

[Veloso et al., 1997] Veloso, M. M, Mulvehill, Alice M., Cox, Michael. Rationale-Supported Mixed-Initiative Case-Based Planning, IAAI Conference Proceedings, 1997.

[Hendler and Mulvehill, 1997] Hendler, J. and Mulvehill, A. High Performance Support for Case-Based Planning Applications, Israeli Conference on Artificial Intelligence, Tel Aviv, Israel, 1996.

[Mulvehill, 1995] Mulvehill, Alice M., Reusing Force Deployment Plans, AAAI 1995 Fall Symposium on Adaptation of Knowledge for Reuse, MIT, Cambridge Mass, 1995.

[Jensen, 1999] Jensen, Jens, Adaptive Course of Action (ACTD), Briefing Charts for ACOA Program, 1999 